\title{
Correction to: Diethylhexyl phthalate (DEHP) regulates the proliferation and chemosensitivity of esophageal squamous cell carcinoma cells via regulation of PTEN
}

\author{
Jian Zhu ${ }^{1} \cdot$ Xuesong Zhang ${ }^{2} \cdot$ Bin Wen ${ }^{4} \cdot$ Jing Chen ${ }^{3} \cdot$ Yuntian $\mathrm{Lu}^{5} \cdot$ Weijun Xu ${ }^{6}$
}

Published online: 15 April 2021

(C) Japan Human Cell Society 2021

\section{Correction to: Human Cell \\ https://doi.org/10.1007/s13577-021-00519-x}

In the original publication of the article, the sixth affiliation should be "Department of Gastroenterology, General Hospital of Eastern Theater of PLA, Nanjing, Jiangsu 210000, People's Republic of China" and the email address of 'Yuntian Lu’ should be 2917565490@qq.com.

Publisher's Note Springer Nature remains neutral with regard to jurisdictional claims in published maps and institutional affiliations.

The original article can be found online at https://doi.org/10.1007/ s13577-021-00519-x.

Weijun Xu

weijunxu@protonmail.com

Jian Zhu

1930560239@qq.com

Xuesong Zhang

16820039@qq.com

Bin Wen

1316000230@qq.com

Jing Chen

15189916888@139.com

Yuntian Lu

2917565490@qq.com

1 Department of Gastroenterology, Jingiiang

People's Hospital, Jingjiang, Jiangsu 214500,

People's Republic of China
2 Department of Central Laboratory, Jingjiang People's Hospital, Jingjiang, Jiangsu 214500, People's Republic of China

3 Department of Pathology, Jingjiang People's Hospital, Jingjiang, Jiangsu 214500, People's Republic of China

4 Department of Oncology, Jingjiang Chinese Medicine Hospital, Jingjiang, Jiangsu 214500, People's Republic of China

5 Department of Bioinformatics, Nantong University Medical School, Nantong, Jiangsu 226001, People's Republic of China

6 Department of Gastroenterology, General Hospital of Eastern Theater of PLA, Nanjing, Jiangsu 210000, People's Republic of China 